\title{
Raman Microspectroscopy of the Yeast Vacuoles
}

\author{
Lucie Bednárová, ${ }^{1}$ Jan Palacký, ${ }^{2}$ Václava Bauerová, ${ }^{1}$ Olga Hrušková-Heidingsfeldová, ${ }^{1}$ \\ Iva Pichová, ${ }^{1}$ and Peter Mojzeš ${ }^{2}$ \\ ${ }^{1}$ Institute of Organic Chemistry and Biochemistry, Academy of Sciences of the Czech Republic, \\ Flemingovo Nám. 2, 16610 Prague 6, Czech Republic \\ ${ }^{2}$ Institute of Physics, Faculty of Mathematics and Physics, Charles University in Prague, \\ Ke Karlovu 5, 12116 Prague 2, Czech Republic
}

Correspondence should be addressed to Lucie Bednárová, bednarova@uochb.cas.cz

Copyright (C) 2012 Lucie Bednárová et al. This is an open access article distributed under the Creative Commons Attribution License, which permits unrestricted use, distribution, and reproduction in any medium, provided the original work is properly cited.

\begin{abstract}
In the present work, real ability of a confocal Raman microspectroscopy to monitor chemical composition of the vacuoles within living yeast cells was investigated and critically assessed. Simple, economical, and practical protocols of the yeast immobilization suitable for less laborious, high-throughput, and spatially resolved Raman measurements were tested for their possible impacts on physiological states and viability of the cells. We have demonstrated that, acquiring Raman spectra from statistically sound sets of immobilized cells and employing advanced multivariate methods for spectral analysis, the chemical composition of the yeast vacuoles can be reliably studied. The most easily and accurately quantifiable seems to be the concentration of polyphosphates which can be unambiguously identified due to unmistakable Raman features. Our approach can be useful for routine, label-free, and noninvasive monitoring of the chemical composition of the vacuoles of living yeasts exposed to various stress factors, the information important in biomedical research of pathogens.
\end{abstract}

Keywords: Raman microspectroscopy, living cell, yeast, vacuole, chemical composition, polyphosphate, Candida albicans

\section{Introduction}

The yeast Candida albicans is an opportunistic human pathogen representing serious threat to immunocompromised individuals. It exhibits considerable metabolic flexibility, which enables the fungus to colonize host niches as diverse as skin, blood, oral cavity, or vaginal mucosa. C. albicans can survive under severe nutrition limitations and escape host immune defense [1]. Recent studies have shown significance of vacuoles for adaptation of $C$. albicans to these changing environments. Vacuoles serve as stores of amino acids, ions, and numerous metabolites. They are involved in $\mathrm{pH}$ homeostasis and osmoregulation, and they play a crucial role in digestion of proteins and recycling of nutrients $[2,3]$. A C. albicans mutant that lacked recognizable vacuoles was not able to kill host macrophages [4]. Information about the chemical composition of the vacuoles within living cells exposed to various external factors could thus be of great importance for development of novel antifungal strategies. 
In the last decade, confocal Raman microspectroscopy has been repeatedly shown as promising contactless and nondestructive method suitable for determination of intracellular chemical composition and chemical imaging of living cells [5]. However, attempts to employ Raman microspectroscopy for routine use in microbiology should cope with numerous technical and methodological problems, for example, adequate fixation assuring both immobility and viability of the cells during exposure to laser beam, compromise between requirements for high spatial resolution and inherent weakness of Raman signal, long acquisition times needed to improve signal-to-noise ratio, discrimination of spectral contributions from cultivation media and supporting materials, acquisition of statistically sound datasets and their treatment, as well as interpretation of complicated Raman spectra in the terms of concentrations of chemical constituents.

In the present work, applicability of the confocal Raman microspectroscopy for determination of chemical composition of the vacuoles within living yeasts was tested and critically assessed. Using both microscope slides and coverslips functionalized by poly-L-lysine, simplified and less laborious immobilization of the yeast cells was achieved. The mounts were found to be suitable for high-throughput, spatially resolved Raman measurements. As the immobilized yeasts immediately stopped their cell cycle due to the washing with water, they remained alive during several hours, and it was possible to acquire Raman spectra from vacuoles of numerous cells. Using advanced multivariate methods [6, 7], mean Raman spectra of the vacuoles throughout the yeast cultures were extracted and compared. Among all specific compounds localized in this compartment, vibrations of polyphosphate appeared to be the most easily and accurately quantifiable characteristic. We show that Raman microspectroscopy can be used in future experiments as a tool to analyze vacuoles of Candida yeasts cultivated under different nutrition conditions, exposed to various stress factors, and/or existing in different physiological states.

\section{Materials and Methods}

The $C$. albicans strain used for this work was clinical isolate HE 169 obtained from the mycological collection of the Faculty of Medicine, Palacky University, Olomouc, Czech Republic. The yeast cells were incubated at $37^{\circ} \mathrm{C}$ in a nutrient-rich YPD medium ( $2 \%$ peptone, $2 \%$ glucose, $1 \%$ yeast extract). Aliquots of the cell suspension were gently centrifuged $(500 \times \mathrm{g}$ for $2 \mathrm{~min})$, washed two times with water, and finally resuspended into the fivefold less amount of water. Few microliters of the suspension were applied to a poly-L-lysine-coated slide, covered with a poly-L-lysine-coated coverslip, and sealed with a clear nail polish. When applying appropriate amount of the cell suspension and gently pressing the coverslip against the slide, combination of the spatial restriction with chemical fixation immobilized the cells in a single layer. The cells were thus in contact with the slide as well as with the coverslip. To eliminate autofluorescence of the glass substrate, fluorescence-free borofloat slides and coverslips (nexterion, schott) were used.

Raman spectra were taken on confocal Raman microspectrometer LabRam HR800 (Horiba) with a He-Ne laser excitation $632.8 \mathrm{~nm}(3-8 \mathrm{~mW}$ at the sample). To obtain well spatially-resolved Raman spectra restricted to the vacuole volume, the 100x oil-immersion objective (NA 1.40) was used in combination with relatively small confocal pinhole $(200 \mu \mathrm{m})$ to eliminate Raman signal from the coverslip and the slide. Raman spectra in the range $500-3900 \mathrm{~cm}^{-1}$ were collected using acquisition time of $60 \mathrm{~s}$ per a cell. Typically, Raman spectra of 50-80 different cells from the same mount were collected. Advanced background- and medium-correction procedures based on singular value decomposition (SVD) methods 
were used for spectral treatment [7]. Statistical evaluation of datasets and visualization of their spectral variability were based on the factor scores provided by SVD analysis. Finally, the spectra were normalized using the band of water $\mathrm{OH}$-stretching vibrations at $\sim 3400 \mathrm{~cm}^{-1}$ as an internal intensity standard.

\section{Results and Discussion}

The main problem in Raman microspectroscopy and imaging of nonadherent living cells is their immobilization. The cells should be attached to the solid surface; however immobilization should not influence negatively their physiological status or spectral measurements. In the case of living cells it is also important that the immobilization procedure is fast and does not represent excessive stress. From that point of view, the protocols relying on the immobilization in polymer matrices (agar, agarose, pectin, gelatin) at elevated temperatures or use of high-viscosity media were found unsuitable for routine Raman experiments due to the risk of thermal shock or spectral contribution from immobilizing medium. As an alternative, we have tried to immobilize Candida at the slides modified by poly-L-lysine. The cells were either left in a cultivation medium or washed by a pure water, dropped on a slide, and observed directly by a water-immersion objective or covered with a coverslip and observed by an oil-immersion objective. However, immobilization by a contact with a single functionalized surface proved to be insufficient for Raman measurements. Even slow motion of the cell during the acquisition, moreover, often escalated by optical tweezers effect of the laser beam completely disrupted spatial resolution. Therefore, combination of the slides and coverslips both functionalized by collagen or poly-L-lysine was tested, the latter being proved to be more efficient. Besides better stabilization due to tight confinement between two functionalized surfaces, higher density of the fixed cells in the field of view and their arrangement into a single layer allowed semiautomated data collection throughout large set of individual cells.

As shown in Figure 1, several yeast intracellular structures can be readily distinguished in the video image; however some of them are of virtually identical shapes and shades, for example, lipidic droplet (E) and round protein-containing structures (C and D). Nevertheless, they can be clearly discerned due to different Raman spectra (Figure 1), without need of staining. Chemical composition of the intracellular structures can be furthermore analyzed in detail, although reliable spectral assignment and decomposition into the spectra of pure constituents could be difficult.

In the case of vacuoles, we have found that Raman spectra can be useful for their identification as well as for analysis of their chemical composition. To average cell-to-cell variability and to extract representative spectrum throughout the cell culture, Raman spectra from vacuoles of different cells have been acquired. Measurements at the same mount producing dozens of Raman spectra did not last more than 1.5 hours in total, the time corresponding to generation time of Candida under the conditions of cultivation. All data collected from the cells cultivated under the similar conditions and supposed to have similar physiological status were treated by the SVD procedure [7] to extract typical Raman spectrum (Figure 1, B), along with representative spectral variability. According to repeated experiments, usually only two orthogonal spectral components were disclosed in datasets, suggesting fair chemical uniformity of the cells in cultures. The cells differ especially in the relative concentration of the main components, not in their absence. The detailed spectral analysis revealed Raman features of amino acids, lipids, and polyphosphates, the principal constituents of Candida vacuoles. In particular the polyphosphates exhibiting typical Raman bands at $\sim 688 \mathrm{~cm}^{-1}$ and $\sim 1154 \mathrm{~cm}^{-1}$ were found in vacuoles at considerably high concentration. We suggest that polyphosphate band at $1154 \mathrm{~cm}^{-1}$ can serve as a marker of the 


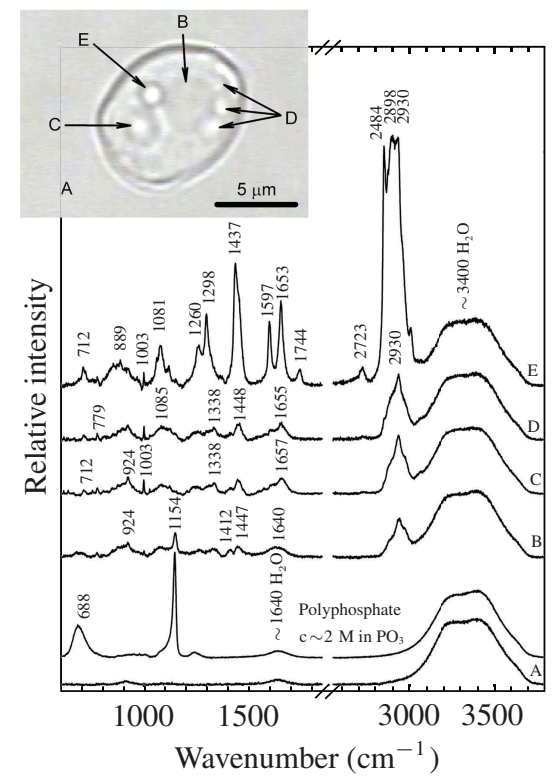

Figure 1: Intracellular structures apparent on the video image of Candida yeast and respective spatiallyresolved Raman spectra of cultivation medium (A), vacuole (B), protein structure I (C), protein structures II (D), and lipidic structure (E). Spectra are normalized using intensity of the water $\mathrm{OH}$-stretching band at $3400 \mathrm{~cm}^{-1}$ as an intensity standard. Raman spectrum of $2 \mathrm{M}$ aqueous solution of polyphosphate is shown to highlight its contribution to the spectrum of vacuole.

vacuoles since it was not detected in the Raman spectra of other Candida compartments. Though the cells coming from the same culture were — at least according to the video image — in a similar physiological status, the absolute concentration of the polyphosphates in their vacuoles was found to vary more than the concentration of other constituents. It was determined quantitatively by means of properly normalized intensity of the $1154 \mathrm{~cm}^{-1}$ band with respect to the intensity of the $\mathrm{OH}$-stretching band of water at $3400 \mathrm{~cm}^{-1}$ (Figure 1). Actual concentration of the polyphosphates in the vacuoles of $C$. albicans cultivated under the standard conditions in YPD medium was found to vary from few dozens up to several hundreds of $\mathrm{mM}$ (expressed as a concentration of $\mathrm{PO}_{3}{ }^{-}$). The differences in the polyphosphate concentration could result from differences in the physiological status of the investigated cell. Raman microspectroscopy thus provides the information that cannot be obtained in situ from living cells by other methods.

\section{Conclusions}

In the present work, immobilization protocol suitable for Raman microspectroscopy of non-adherent cells was tested. It was demonstrated that with the properly immobilized Candida cells it is possible to collect spatially-resolved Raman spectra of individual compartments within living cells. The spectra can serve for unambiguous identification of intracellular compartments of similar appearance as well as for investigation of their chemical content. We report that the yeast vacuoles can be reliably 
identified by the polyphosphate Raman bands at 688 and $1154 \mathrm{~cm}^{-1}$, the spectral features missing in other yeast compartments. Using polyphosphate Raman bands normalized with respect to the water signal, actual polyphosphate concentration can be measured inside the vacuoles of living cells. Raman microspectroscopy will be furthermore employed for investigation of the $C$. albicans vacuoles and their chemical content affected by nutrition, stress, or phase of cell cycle conditions.

\section{Acknowledgments}

Ministry of Education of the Czech Republic (MSM0021620835), the Grant Agency of the Czech Republic (P208/10/0376 and 310/09/1945), and Research Project Z 40550506 are acknowledged for financial support.

\section{References}

[1] J. Kim and P. Sudbery, "Candida albicans, a major human fungal pathogen," Journal of Microbiology, vol. 49, no. 2, pp. 171-177, 2011.

[2] S. C. Li and P. M. Kane, "The yeast lysosome-like vacuole: endpoint and crossroads," Biochimica et Biophysica Acta, vol. 1793, no. 4, pp. 650-663, 2009.

[3] V. Veses, A. Richards, and N. A. Gow, "Vacuoles and fungal biology," Current Opinion in Microbiology, vol. 11, no. 6, pp. 503-510, 2008.

[4] G. E. Palmer, M. N. Kelly, and J. E. Sturtevant, "The Candida albicans vacuole is required for differentiation and efficient macrophage killing," Eukaryotic Cell, vol. 4, no. 10, pp. 1677-1686, 2005.

[5] M. Diem, M. Romeo, S. Boydston-White, M. Miljković, and C. Matthäus, "A decade of vibrational micro-spectroscopy of human cells and tissue (1994-2004)," Analyst, vol. 129, no. 10, pp. 880-885, 2004.

[6] E. R. Malinowski, Factor Analysis in Chemistry, Wiley, New York, NY, USA, 2002.

[7] J. Palacký, P. Mojzeš, and J. Bok, "SVD-based method for intensity normalization, background correction and solvent subtraction in Raman spectroscopy exploiting the properties of water stretching vibrations," Journal of Raman Spectroscopy, vol. 42, no. 7, pp. 1528-1539, 2011. 


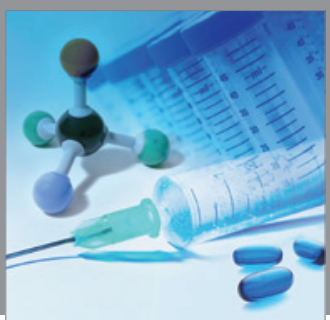

International Journal of

Medicinal Chemistry

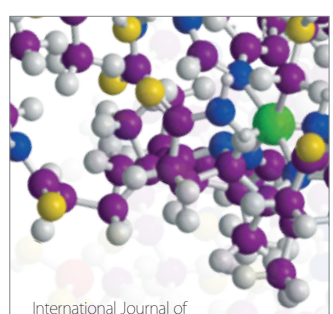

Carbohydrate Chemistry

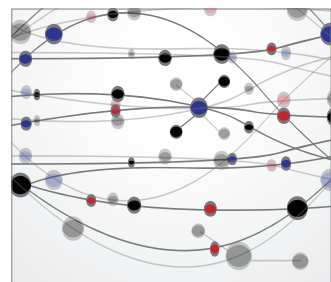

The Scientific World Journal
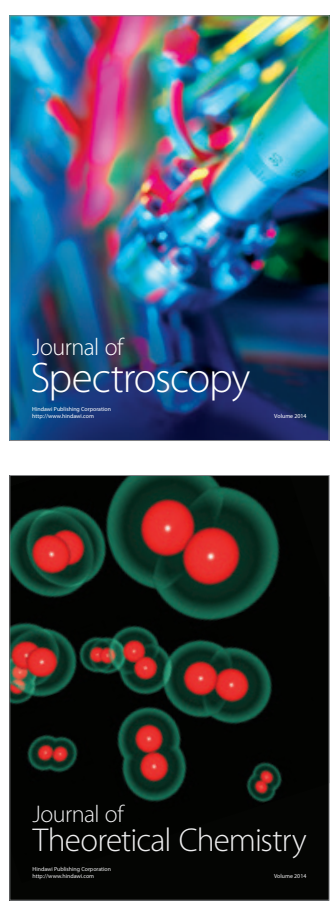
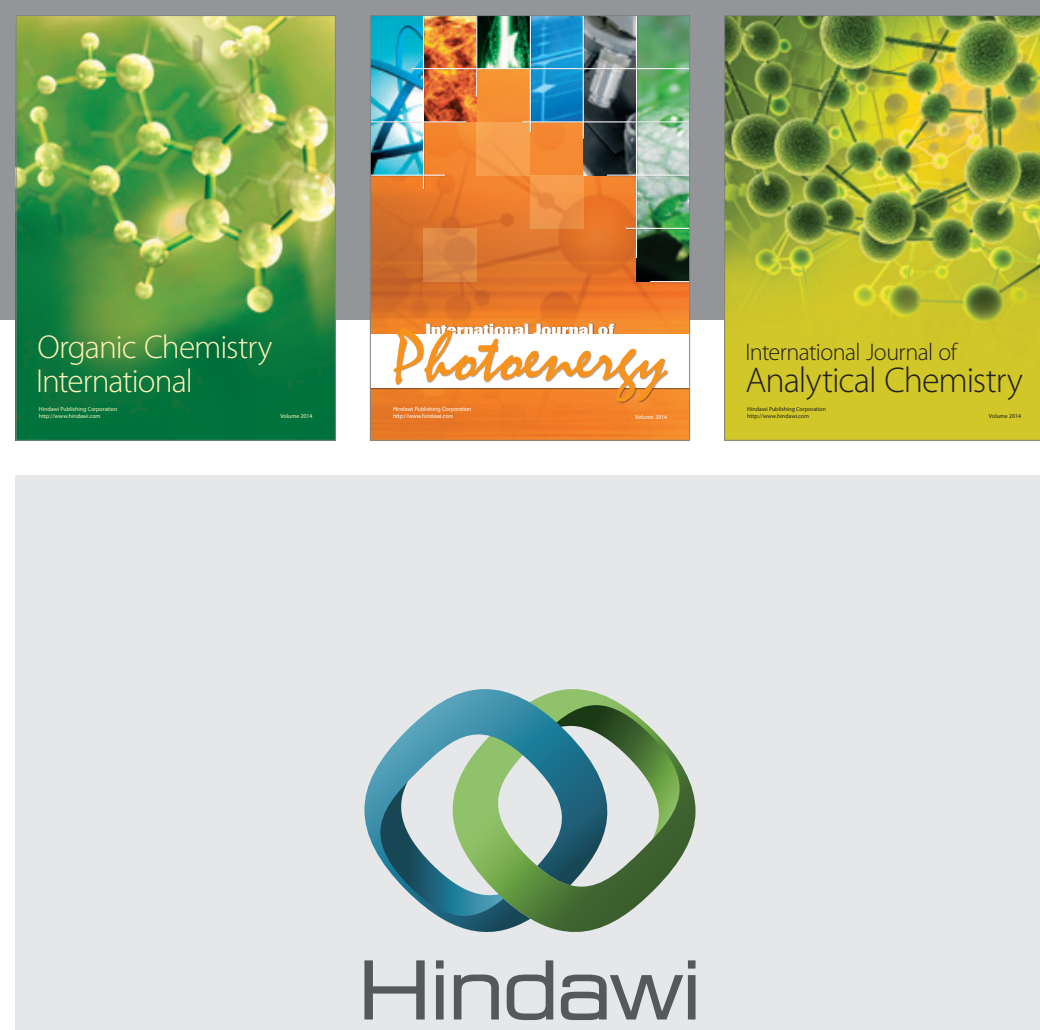

Submit your manuscripts at

http://www.hindawi.com
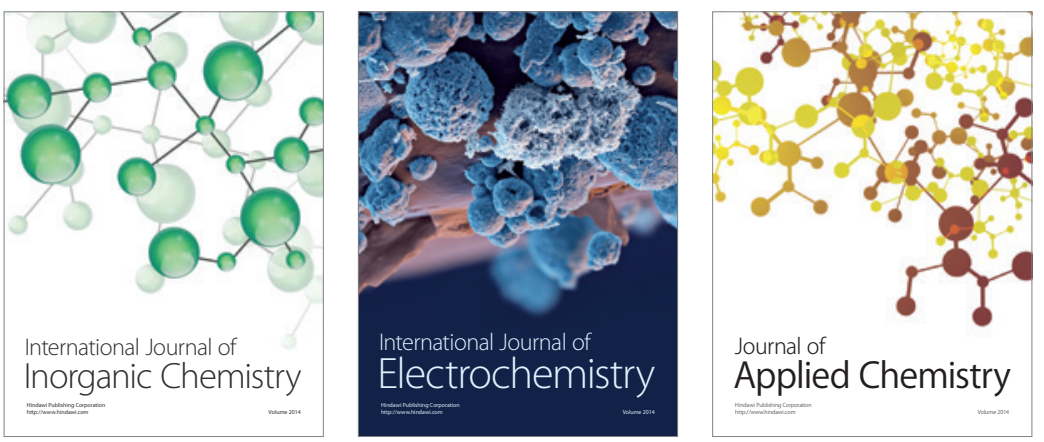

Journal of

Applied Chemistry
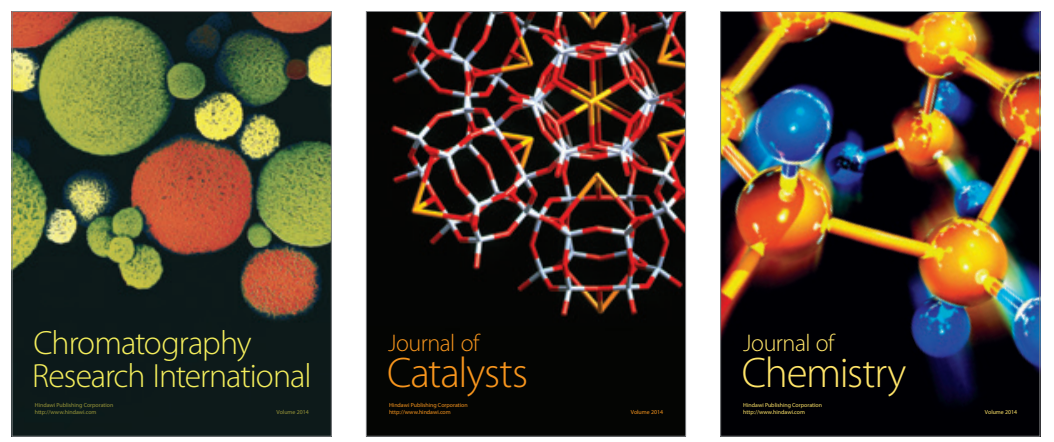
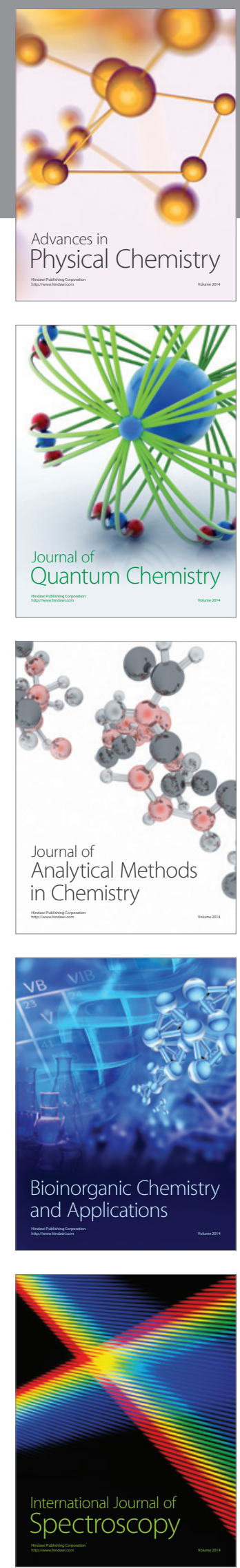